\title{
Acute pancreatitis in children
}

\author{
Urszula Grzybowska-Chlebowczyk ${ }^{1}$, Martyna Jasielska², Anna Flak-Wancerz², Sabina Więcek², \\ Katarzyna Gruszczyńska3 ${ }^{3}$, Wojciech Chlebowczyk ${ }^{4}$, Halina Woś ${ }^{1}$
}

${ }^{1}$ Department of Paediatrics, School of Medicine, Medical University of Silesia, Katowice, Poland 2Department of Gastroenterology, Upper-Silesian Child Health Care Centre, Katowice, Poland

${ }^{3}$ Department of Diagnostic Imaging and Interventional Radiology, School of Medicine, Medical University of Silesia, Katowice, Poland

${ }^{4}$ Department of Nursing and Social Medical Problems, School of Health Sciences, Medical University of Silesia, Katowice, Poland

Key words: acute pancreatitis, children, paediatric, aetiology.

Address for correspondence: Urszula Grzybowska-Chlebowczyk MD, Department of Paediatrics, Gastroenterology Unit Upper-Silesian Child Health Care Centre, Medical University of Silesia, 16 Medyków St, 40-752 Katowice, Poland, phone: +48 322071700 , fax: +48 3220717 21, e-mail: klinikapediatrii@sum.edu.pl

\begin{abstract}
Introduction: The acute pancreatitis is a rare disease, but it has started to be diagnosed more often in children.

Aim: The aim of the study was single-centre, retrospective analysis of the incidence, aetiology, and clinical course of acute pancreatitis in children.

Material and methods: We analysed the medical records of patients with acute pancreatitis hospitalised in the Gastroenterology Unit of the Paediatrics Department, Medical University of Silesia from Jan 2004 to Dec 2013.

Results: There were 76 cases of acute pancreatitis among 51 children (average age: 12.07 years) hospitalised in the Gastroenterology Unit between January 2004 and December 2013. The diagnosis of acute pancreatitis was performed on the basis of INSPIRE criteria and modified Atlanta classification. Patients were divided into groups: I - 1-12 years old, which included $20(39.21 \%)$ children and II - 13-18 years old, with $31(60.78 \%)$ children. The idiopathic aetiology was the most common cause of acute pancreatitis, occurring in 22 (43.1\%) children, and in 15 cases the aetiology of the disease was biliary (29.4\%). Genetically determined causes were diagnosed in 8 (15.7\%) patients, the PRSS1 mutation in four patients, mutation in SPINK 1 in 1 child, and CFTR gene mutation in 1 child. Two children simultaneously had two genes mutations (CFTR, SPINK 1 ), and during the considered period had more than one episode of acute pancreatitis.

Conclusions: Acute pancreatitis of idiopathic aetiology was most common among the examined children, and this should encourage the continued search for the causes of disease, especially genetic, and with particular emphasis on younger age group.
\end{abstract}

\section{Introduction}

Acute pancreatitis (AP) is a disease in which there is an autolysis of this organ. Inflammatory changes in the pancreas and its surroundings are a consequence of the release of the enzymatic cascade initiated by the conversion of trypsinogen to trypsin. There are two pathological forms of AP: pancreatic oedema, with rather mild course and pancreatic or peripancreatic necrosis, with a poor prognosis, but rare in childhood (less than $1 \%$ ).

The aetiology, clinical course, and treatment of pancreatitis are different in children and adults. However, so far to recognise the AP and in clinical practice, the Atlanta modified guidelines were used by extrapolat- ing them to the patient population of children under 18 years of age [1, 2]. It was not until 2012 when the group of experts published the INSPPIRE (International Study Group of Paediatric Pancreatitis: In search of a cURE) guidelines, the aim of which was to establish the definition of AP, acute recurrent pancreatitis, and chronic pancreatitis in children [3]. According to INSPPIRE, AP can be recognised when there are two of the following three criteria: clinical symptoms, particularly abdominal pain, which suggests pancreatitis (abdominal pain of acute onset), nausea, vomiting, and back pain; increased levels of serum amylase and/or lipase at least three times greater than the upper limit of normal and imaging findings characteristic for AP. Acute 
recurrent pancreatitis requires at least two episodes of AP separated by at least a 1-month pain-free interval or complete normalisation of serum pancreatic enzyme levels and pain-free period, irrespective of a specific time interval between AP episodes.

Acute pancreatitis is a rare disease, but it has started to be diagnosed more often in children. The incidence is estimated at 3.6-13.2/100,000 per year [4, 5]. The reasons for increased incidence of AP can be different and are not fully understood, but it is considered that they may be associated with a greater awareness of the disease in the paediatric population [6-8].

\section{Aim}

The aim of the study was a single-centre, retrospective analysis of the incidence, aetiology, and clinical course of AP in children.

\section{Material and methods}

We retrospectively analysed the medical records of patients with acute pancreatitis hospitalised in the Gastroenterology Unit of the Department of Paediatrics Medical University of Silesia within 10 years - from January 2004 to December 2013. There were 76 cases of AP in 51 children. The diagnosis of AP was made based on the INSPIRE criteria and modified Atlanta criteria (two of three criteria) - a threefold increase in biochemical markers (amylase or lipase), characteristic abdominal pain, and changes in imaging examinations [1-3].

The following data were analysed: the aetiology of AP, the basic values of biochemical parameters (amylase and lipase in blood serum, concentration of the alanine aminotransferase, gamma-glutamyltranspeptidase, total bilirubin, C-reactive protein, levels of leukocytes in the blood), imaging findings (ultrasonography, computed tomography and magnetic resonance imaging of the abdomen), and the length of hospitalisation.

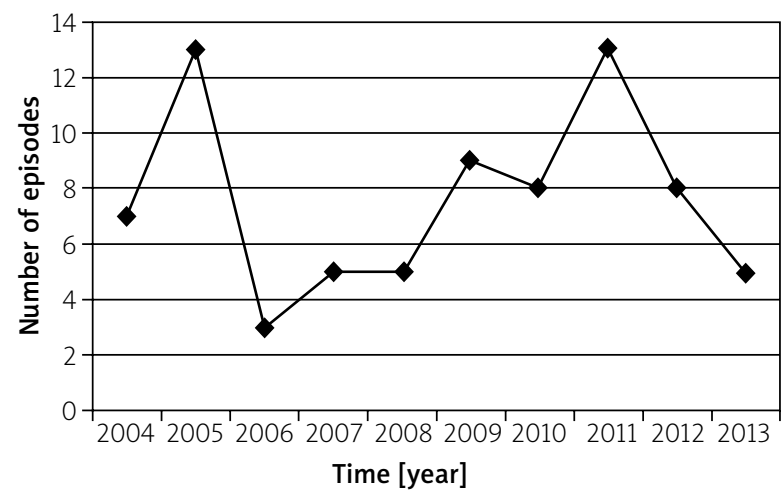

Figure 1. The incidence of acute pancreatitis in subsequent years

\section{Statistical analysis}

The data were subjected to statistical analysis using Statistica 10.0. All analyses were performed with a 95\% significance interval. Due to the qualitative nature of the variables, the sample size $n>40$, and the occurrence of expected size $<10$, the $\chi^{2}$ test for qualitative variables $\mathrm{V}^{2}$ was used.

\section{Results}

\section{The incidence of acute pancreatitis}

Seventy-six cases of AP among 51 children (53\% girls - 27 children) were reported. The youngest child was 20 months old, the oldest was 18 years old, and the average age was 12.07 years. Patients were divided into two age groups: I - 1-12 years, which included $20(39.21 \%)$ children and II - 13-18 years, with 31 $(60.78 \%)$ children. In the younger age group 10 children were under 7 years of age.

The incidence of AP in subsequent years is shown in Figure 1.

\section{Aetiology}

The most common aetiology of AP was idiopathic - 43.1\%. Biliary aetiology occurred in 15 (29.4\%) children, among them in one child, during imaging examination, anomalies in the form of choledochal cyst were found and one patient with congenital spherocytosis with extrahepatic and intrahepatic cholestasis was reported. Genetically determined causes were diagnosed in $8(15.7 \%)$ patients, the mutation in the trypsinogen (PRSS1) in 4 children, mutation in the gene encoding trypsin inhibitor (SPINK 1) in 1 child, and mutation in the CFTR gene in 1 child. It is worth noting that 2 children simultaneously had two gene mutations (CFTR and SPINK 1), and during the considered period 1 boy had 5 episodes of AP, and the second one - two. Isolated cases of pancreatitis caused by separate factors were recognised as other causes of AP. This group included patients with AP due to the following reasons: iatrogenic - after endoscopic retrograde cholangiopancreatography (ERCP) in 1 child; occurring with the first appearance of ulcerative colitis; alcoholic - in an almost 18-yearold patient with a history of alcohol abuse directly before the onset of symptoms; and toxic - in a 2-year-old patient due to consumption of the petroleum ether. In 2 children post-traumatic recurrent AP was diagnosed, among them in 1 child, 3 months earlier a classic bicycle handlebars injury occurred, after which AP occurred twice (after the second episode - large cyst in the pancreatic tail) (Table I).

In the younger age group the idiopathic aetiology of pancreatitis dominated (21.6\%), while among older 
Table I. The causes of acute pancreatitis

\begin{tabular}{lc} 
Acute pancreatitis aetiology & $\boldsymbol{N}(\%)$ \\
\hline Biliary: & $15(29.4)$ \\
\hline Cholecystolithiasis & $13(25.4)$ \\
\hline Biliary tract anomalies & $1(2)$ \\
\hline Hereditary spherocytosis & $1(2)$ \\
\hline Genetic: & $8(15.8)$ \\
\hline PRSS1 & $4(7.8)$ \\
\hline SPINK1 & $1(2)$ \\
\hline CFTR & $1(2)$ \\
\hline Mutations in two genes (SPINK1, CFTR) & $2(3.9)$ \\
\hline Idiopathic & $22(43.1)$ \\
\hline Other & $6(11.7)$
\end{tabular}

children AP of idiopathic cause ( $21.6 \%$ children) and biliary cause (21.6\%) occurred equally often (Figure 2 ).

In the analysed period, $12(23.4 \%)$ children were hospitalised more than once due to AP (Table II). Another episode of AP in the younger age group occurred in $8(15.6 \%)$ children, and in the older group in 4 (7.8\%) patients. Most frequently, disease recurrence was in cases of AP of idiopathic cause.

\section{Clinical symptoms}

The most frequently reported clinical symptom of AP was abdominal pain, which occurred in 73 cases of

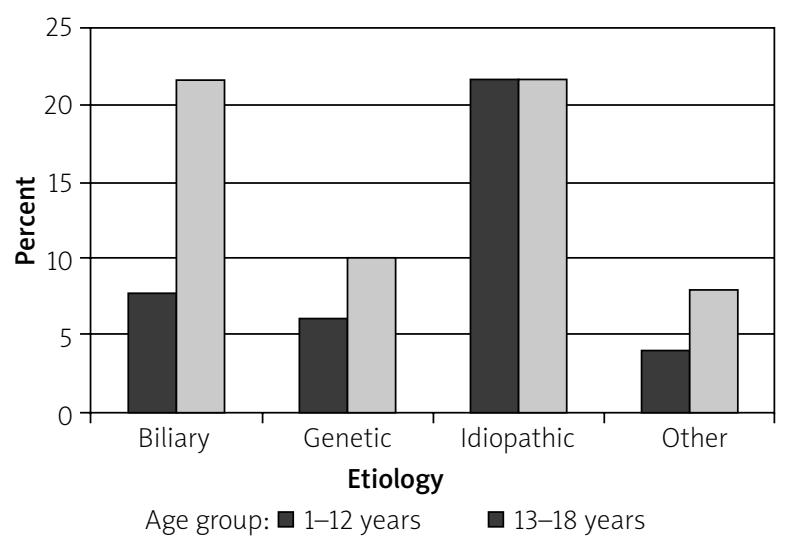

Figure 2. The aetiology of acute pancreatitis in different age groups

disease (96\%), and vomiting was observed in $40.8 \%$ of children. Fever was a rare symptom, concerning only $3.8 \%$ of patients. There was no statistically significant relationship between occurrence of symptoms and the age of patients $(p>0.05)$.

\section{Results of laboratory tests}

The increased levels of amylase enzyme activity in serum greater than three upper limits of normal were found in 63 (82.9\%) cases of AP, of which $32.9 \%$ exceeded tenfold. In 28 children the serum lipase activity was investigated, in 19 children the level was least three times greater than the upper limit of normal, and in 3 patients the result was positive, with normal amylase activity in serum. The concentration of C-re-

Table II. Characteristics of children with recurrent acute pancreatitis

\begin{tabular}{|c|c|c|c|c|}
\hline Case & Gender & $\begin{array}{l}\text { Number of episodes during } \\
\text { the considered period }\end{array}$ & $\begin{array}{l}\text { Age at the time of } \\
\text { I episode [years] }\end{array}$ & Aetiology \\
\hline 1 & M & 5 & 10 & Idiopathic \\
\hline 2 & M & 5 & 6 & Mutations in the SPINK1 and CFTR gene \\
\hline 3 & $\mathrm{~F}$ & 5 & 4 & Mutation in the PRSS1 gene \\
\hline 4 & $\mathrm{~F}$ & 4 & 3 & Idiopathic \\
\hline 5 & M & 4 & 12 & Idiopathic \\
\hline 6 & $\mathrm{~F}$ & 2 & 12 & Mutation in the PRSS1 gene \\
\hline 7 & $\mathrm{~F}$ & 2 & 15 & Episodes associated with ulcerative colitis \\
\hline 8 & M & 2 & 4 & Idiopathic \\
\hline 9 & $\mathrm{~F}$ & 2 & 16 & Mutation in the SPINK1 gene \\
\hline 10 & $\mathrm{~F}$ & 2 & 17 & Idiopathic \\
\hline 11 & M & 2 & 12 & Mutations in the SPINK1 and CFTR gene \\
\hline 12 & $M$ & 2 & 17 & Idiopathic \\
\hline
\end{tabular}


Table III. The presence of laboratory tests depending on the aetiology of acute pancreatitis

\begin{tabular}{|c|c|c|c|c|c|}
\hline \multirow{2}{*}{$\begin{array}{l}\text { Aetiology of acute } \\
\text { pancreatitis }\end{array}$} & \multicolumn{4}{|c|}{ Blood laboratory tests } & \multirow[t]{2}{*}{$P$-value } \\
\hline & $\begin{array}{l}\text { Amylase } \\
>3 \times N\end{array}$ & $\begin{array}{l}\text { Leukocytosis } \\
>12 \times 10^{3} / \mu \mathrm{l}\end{array}$ & $\begin{array}{c}\text { C-reactive protein } \\
>10 \mathrm{mg} / \mathrm{l}\end{array}$ & $\begin{array}{c}\text { Total bilirubin } \\
>17 \mu \mathrm{mol} / \mathrm{l}\end{array}$ & \\
\hline Idiopathic & $13(25.5 \%)$ & $6(11.8 \%)$ & $9(17.7 \%)$ & $8(15.6 \%)$ & $>0.05$ \\
\hline Biliary & $18(35.3 \%)$ & $5(9.8 \%)$ & $7(13.7 \%)$ & $0(0 \%)$ & \\
\hline Genetic & $6(9.8 \%)$ & $3(5.9 \%)$ & $4(7.8 \%)$ & $0(0 \%)$ & \\
\hline Other & $5(9.8 \%)$ & $0(0 \%)$ & $1(2 \%)$ & $0(0 \%)$ & \\
\hline
\end{tabular}

Amylase N: 25-125 U/l.

active protein was increased in 29 (38.1\%) children, whereas leukocytosis was observed in 25 (32.9\%) patients (Table III). Hiperbilirubinaemia occurred in 8 children and was observed only in children with biliary aetiology, which was statistically significant $(p<0.05)$. There were no statistically significant $a b-$ normalities in other laboratory studies depending on the age and aetiology of AP.

\section{The results of imaging}

All patients underwent abdominal ultrasound examination. In 51 (67.1\%) cases of AP characteristic pancreatic parenchyma changes in the form of swelling, changes in echogenicity, or presence of peripancreatic fluid collections were shown. Imaging examinations were extended by the computed tomography (CT) scan of the abdomen in 56 cases of AP. Among performed examinations, in $60 \%$ of cases changes specific to pancreatitis occurred. The oedema pancreatitis was diagnosed in 29 patients, necrotising pancreatitis in 5 children. In 13 children cholecystitis, gallstones, or cholelithiasis biliary were diagnosed (Figure 3).

Magnetic resonance imaging cholangiography was performed in 23 (45\%) children; choledocholitiasis was diagnosed in $5(9.8 \%)$ children, cholelithiasis in $4(7.8 \%)$ children, and in one girl the biliary cysts type IV occurred.

\section{Hospitalisation}

The hospitalisation period ranged from 4 to 48 days (average: 13.8 days). Eight (10.5\%) patients were discharged within 7 days, and hospitalisation for more than 2 weeks involved 27 (35.5\%) children. The median was 13 days. In age group I (1-12 years) $21.6 \%$ of the children stayed on the ward for less than 14 days and $15.7 \%$ for more than 14 days. There was no death of a patient due to AP or its complications.

The longest hospitalisation (over 14 days) occurred in children with the AP of biliary aetiology - 10 (19.6\%) patients, and the shortest, to 7 days, in patients with
AP of unknown aetiology and AP from other causes 4 (7.8\%). The statistical significance between the length of hospitalisation and the age and aetiology of AP had not been demonstrated $(p>0.05)$.

\section{Treatment}

Five children were qualified for therapeutic endoscopic retrograde cholangiopancreatography (ERCP), which took place in the Department of Gastroenterology of the Medical University of Silesia. Six (11.7\%) patients were transferred to the Department of Surgery to surgical treatment: 2 children with pancreatic cysts, 2 because of cholelithiasis, and 1 child because of both cholelithiasis and pancreatic cyst. One girl (13 years old) with biliary AP was transferred because of numerous cysts of the pancreas; additionally, in the CT image thrombosis of the portal vein was visualised. The other children were treated conservatively.

\section{Discussion}

In recent years, many studies have drawn attention to the increasing incidence of AP in both adults and children. This phenomenon was described for the first time by Lopez in a children's hospital in Dallas (from 5 to 113 patients/years from 1993 to 1998), which has been confirmed by other researchers from various parts of the world [4, 5, 9-12]. In our 10-year study, the incidence of AP was between 3 and 13 episodes per year, the increasing trend of this disease incidence was not confirmed. On the other hand, the recurrence of AP occurred in $23.4 \%$ of children. In the literature the described frequency of relapse of AP is different: Kandula and Lowe describe 10\%, Park - 15\%, Chen - $21.3 \%$, and Sanchez- Ramirez - 34.5\% [11, 13-15].

Among our patients the biliary aetiology involved nearly $30 \%$ of cases of AP, and it was also one of the dominant causes of AP among children from the older age group. It is consistent with previous studies, in which cholelithiasis was the cause of $10-30 \%$ of pancreatitis in children $[11,13,14,16,17]$. In other studies, 

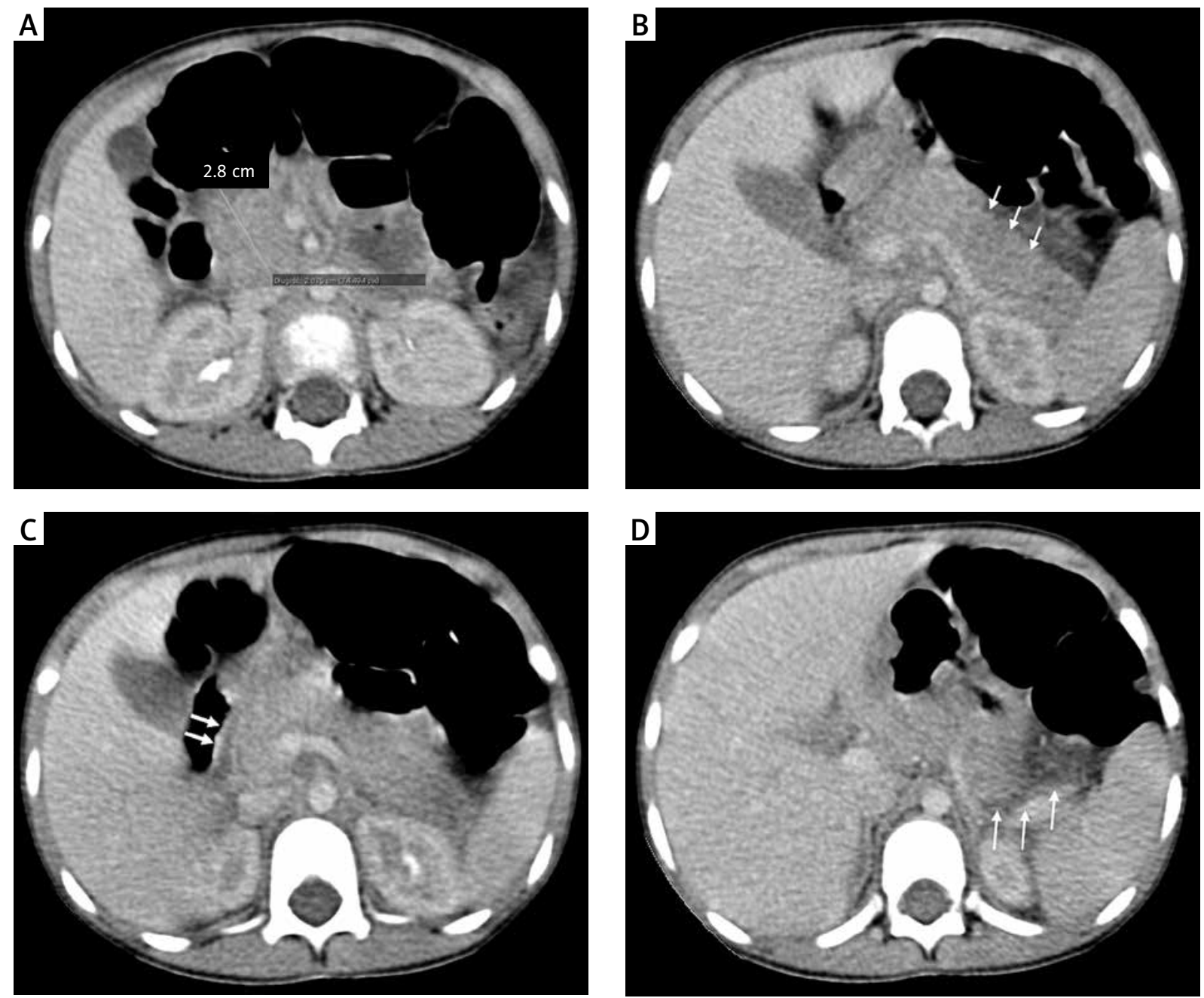

Figure 3. Computed tompography in a girl with acute pancreatitis. A - Enlarged pancreatic head. B - Features of swelling of the pancreas tail (arrows). C - Fluid in the retroperitoneal space in the vicinity of the pancreatic head (arrows). D - Fluid in the retroperitoneal space in the front of the renal fascia (arrows)

similar to our work, the frequency of biliary aetiology was higher among older children [11]. Acute pancreatitis of idiopathic aetiology was diagnosed in $43.1 \%$ of children, which is slightly higher than reported in the literature, where the frequency is described at the level of $8 \%$ (Werlin) to $34 \%$ (DeBanto) $[4,9,14]$. It is worth mentioning that the idiopathic aetiology most frequently involved younger children. The third leading cause of AP was genetic mutations (in the SPINK 1, PRSS1, and CFTR genes) found among almost $16 \%$ of children, including 2 children with two mutations, and these children had more than one episode of pancreatitis in the analysed period. The frequency of this pancreatitis reason is described in the literature at the level of $2.5-9.8 \%$ [9, 17] In 2012, the Sultan showed a high prevalence of genetic mutations (in the SPINK, PRSS 1, and CFTR genes) in patients with recurrent and chronic pancreatitis without other causes of these diseases (anatomic and metabolic) [18]. It is known that mutations in the gene PRSS 1 (two types: $\mathrm{R} 122 \mathrm{H}$ and N29I) are responsible for hereditary pancreatitis, and SPINK 1 gene mutation is strongly associated (about $20 \%$ of patients) with idiopathic chronic pancreatitis in children and adolescents [19]. In recent years, some other mutations associated with the early occurrence of acute and chronic pancreatitis have been described - mutations in the CPA1 gene encoding carboxypeptidase $A 1$ (one of the pancreatic metalloproteinases) [20]. In Poland, SPINK 1, PRSS1, CFTR, and AAT gene mutations were examined by Sobczyńska-Tomaszewska et al. They obtained the highest incidence of mutations in the field of SPINK 1 and PRSS 1 genes among patients with chronic pancreatitis and recurrent AP, and among members of the families of these patients [21]. In our 
study, we examined mutations only in three genes, but it is worth noting that in 2 children with recurrent AP and a positive family history of recurrent AP the cause of the disease was not found (also a genetic mutation). It seems that in some children, who did not show the cause of AP, the mutation could occurred in other genes than in the three tested by us.

Among our patients, the median hospitalisation was 13 days, and the average was 13.8 days, children from the younger age group most often were hospitalised for $7-14$ days ( $21.6 \%$ of children), and patients from the older age group - over 14 days (29.4\% of patients). However, this is shorter than in other studies; the average length of hospitalisation described by Werlin et al. was 24 days, and by Sanchez-Ramirez et al. was 25.7 days. This is probably related with the overwhelming number of children without any complications in our study $[12,15]$. However, the median hospitalisation of our patients was longer than described by other authors - Park et al. (5 days) and Werlin et al. (8 days) $[11,12]$.

The most common symptoms among the studied children were the abdominal pain and vomiting (96\% and $40 \%$ of children), which is confirmed by previous research $[11,12,14]$. Fever was observed in a small percentage of children (3.8\%), in contrast to some other researchers. Kandula and Lowe [13] described fever in $40 \%$ of patients, Chen et al. in 33\% [14], and SanchezRamirez et al. in $27 \%$ [15].

Laboratory indicators of pancreatitis are the serum amylase and/or lipase activity at least three times greater than the upper limit of normal. Increased amylase activity was recorded in $83 \%$ of patients, just like in research from the UK and the US [12, 22]; a little lower (about $50 \%$ of patients) was described by Park et al. [11]. In 3 children amylase activity was within normal limits but the lipase enzyme activity was increased; these children were between the ages of 8 to 13 years. It is not rare because it is estimated that approximately $20 \%$ of patients with pancreatitis have normal amylase levels [7]. At the same time, Park and Kandula pointed out that the measurement of lipase activity might be a more sensitive indicator of AP than amylase among younger patients (100\% vs. $40-60 \%)$ $[11,13]$. Moreover, Park et al. in their study proved that a 1.5 -fold increase in lipase activity above the upper limit of normal in young children might be associated with the occurrence of AP [11].

All children had an abdominal ultrasound examination, and characteristic changes were observed in $67 \%$ of patients, slightly more than in the Werlin et al. and Sanchez-Ramirez et al. study [12, 15]. Computed tomography scan of the abdomen was performed in $73.6 \%$ of children, of which $60 \%$ visualised lesions characteristic for AP. Additionally, some researchers have shown that in the younger group of children the frequency of $\mathrm{CT}$ is lower (about 60\%), which is related to saving children from exposure to ionising radiation and relatively low sensitivity of this test $[13,23]$. According to guidelines from the Atlanta and INSPIRE group of experts, if there are two criteria of AP, i.e. characteristic abdominal pain and three times greater than the upper limit of normal pancreatic enzymes activity, there is no need to perform $\mathrm{CT}$ of the pancreas [1-3]. Computed tomography is not recommended, especially in the first phase of pancreatitis (oedematous); however, it is recommended when pancreatic necrosis is suspected $[1,2,6]$. The sensitivity of CT for the diagnosis of AP is estimated at $47-81 \%$ $[11,13,14]$. In recent years increasing interest in the prognostic significance of CT in children with severe AP can be seen [24-26]. In 2012 Lautz et al. assessed the predictive value of the CTSI scale (Computed Tomography Severity Index), used in adults, in a series of 64 children with AP. CTSI showed greater predictive value (sensitivity $81 \%$, specificity $76 \%$, PPV $62 \%$, and NPV 90\%) in predicting severe AP and serious clinical complications in relation to Ransons scale, the AP scale, and the scale of Glasgow [23]. However, so far a prospective study documenting the value of the CTSI in children with AP has not been carried out.

\section{Conclusions}

Acute pancreatitis of idiopathic aetiology was the most common among the examined children, and this fact should encourage researchers to continue looking for the causes of disease, especially genetic, and with particular emphasis on the younger age group. Due to the limited number of examined children in the available, current literature, it seems necessary to plan a large, prospective, multi-centre study in order to evaluate the incidence, aetiology, natural history, and evaluation of the effectiveness of available prognostic scales of AP.

\section{Conflict of interest}

The authors declare no conflict of interest.

\section{References}

1. Bradley EL. A clinically based classification system for acute pancreatitis. Summary of the International Symposium on Acute Pancreatitis, Atlanta, Ga, September 11 through 13, 1992. Arch Surg 1993; 128: 586-90.

2. Banks PA, Bollen TL, Dervenis C, et al. Classification of acute pancreatitis--2012: revision of the Atlanta classification and definitions by international consensus. Gut 2013; 62: 102-11. 
3. Morinville VD, Husain SZ, Bai $\mathrm{H}$, et al. Definitions of pediatric pancreatitis and survey of present clinical practices. J Pediatr Gastroenterol Nutr 2012; 55: 261-5.

4. Nydegger A, Heine RG, Ranuh R, et al. Changing incidence of acute pancreatitis: 10 -year experience at the Royal Children's Hospital, Melbourne. J Gastroenterol Hepatol 2007; 22: 1313-6.

5. Morinville VD, Barmada MM, Lowe ME. Increasing incidence of acute pancreatitis at an American pediatric tertiary care center: is greater awareness among physicians responsible? Pancreas 2010; 39: 5-8.

6. Bai HX, Lowe ME, Husain SZ. What have we learned about acute pancreatitis in children? J Pediatr Gastroenterol Nutr 2011; 52: 262-70.

7. Abu-El-Haija M, Lin TK, Palermo J. Update to the management of pediatric acute pancreatitis: highlighting areas in need of research. J Pediatr Gastroenterol Nutr 2014; 58: 689-93.

8. Lowe ME. Pancreatitis in childhood. Curr Gastroenterol Rep 2004; 6: 240-6.

9. DeBanto JR, Goday PS, Pedroso MR, et al. Acute pancreatitis in children. Am J Gastroenterol 2002; 97: 1726-31.

10. Lopez MJ. The changing incidence of acute pancreatitis in children: a single-institution perspective. J Pediatr 2002; 140: 622-4.

11. Park A, Latif SU, Shah AU, et al. Changing referral trends of acute pancreatitis in children: a 12-year single-center analysis. J Pediatr Gastroenterol Nutr 2009; 49: 316-22.

12. Werlin SL, Kugathasan S, Frautschy BC. Pancreatitis in children. J Pediatr Gastroenterol Nutr 2003; 37: 591-5.

13. Kandula L, Lowe ME. Etiology and outcome of acute pancreatitis in infants and toddlers. J Pediatr 2008; 152: 106-10, 10.e1.

14. Chen CF, Kong MS, Lai MW, Wang CJ. Acute pancreatitis in children: 10-year experience in a medical center. Acta Paediatr Taiwan 2006; 47: 192-6.

15. Sánchez-Ramírez CA, Larrosa-Haro A, Flores-Martínez S, et al. Acute and recurrent pancreatitis in children: etiological factors. Acta Paediatr 2007; 96: 534-7.

16. Antunes H, Nascimento J, Mesquita A, Correia-Pinto J. Acute pancreatitis in children: a tertiary hospital report. Scand J Gastroenterol 2014; 49: 642-7.

17. Minen F, De Cunto A, Martelossi S, Ventura A. Acute and recurrent pancreatitis in children: exploring etiological factors. Scand J Gastroenterol 2012; 47: 1501-4.

18. Sultan M, Werlin S, Venkatasubramani N. Genetic prevalence and characteristics in children with recurrent pancreatitis. J Pediatr Gastroenterol Nutr 2012; 54: 645-50.

19. Räty S, Piironen A, Babu M, et al. Screening for human cationic trypsinogen (PRSS1) and trypsinogen inhibitor gene (SPINK1) mutations in a Finnish family with hereditary pancreatitis. Scand J Gastroenterol 2007; 42: 1000-5.

20. Witt $\mathrm{H}$, Beer S, Rosendahl J, et al. Variants in CPA1 are strongly associated with early onset chronic pancreatitis. Nat Genet 2013; 45: 1216-20. Haddock G, Coupar G, Youngson GG, et al. Acute pancreatitis in children: a 15-year review. J Pediatr Surg 1994; 29: 719-22.

21. Sobczyńska-Tomaszewska A, Bak D, Oralewska B, et al. Analysis of CFTR, SPINK1, PRSS1 and AAT mutations in children with acute or chronic pancreatitis. J Pediatr Gastroenterol Nutr 2006; 43: 299-306.
22. Park AJ, Latif SU, Ahmad MU, et al. A comparison of presentation and management trends in acute pancreatitis between infants/toddlers and older children. J Pediatr Gastroenterol Nutr 2010; 51: 167-70.

23. Lautz TB, Turkel G, Radhakhrishnan J, et al. Utility of the computed tomography severity index (Balthazar score) in children in acute pancreatitis. J Paediatric Surg 2012; 47: 1185-91.

24. Rainzer MD, Phatak UP, Baker K, et al. Acute necrotizing pancreatitis in children. J Pediatr 2013; 162: 788-92.

25. Suzuki M, Saito N, Naritaka N, et al. Scoring system for prediction of acute pancreatitis in children. Pediatr Int 2015; 57 : 113-8.

26. Fabre A, Petit P, Gaudart J, et al. Severity scores in children with acute pancreatitis. J Pediatr Gastroenterol Nutr 2012; 55 : 266-7.

Received: 21.09.2015

Accepted: 21.03.2016 